\title{
A PROBLEM OF SALLEE ON EQUIDECOMPOSABLE CONVEX BODIES ${ }^{1}$
}

\author{
R. J. GARDNER
}

\begin{abstract}
We show that equidecomposable planar convex bodies need not be convex equidecomposable. This answers a question of Sallee. We also show that convex and scissors equidecomposability are equivalent notions for convex bodies in the plane, and include a discussion of these concepts in higher dimensions.
\end{abstract}

The Wallace-Bolyai-Gerwien theorem, proved in the last century, says that if polygons $A$ and $B$ are of equal area, polygon $A$ may be cut into triangles which may then be rearranged to form polygon $B$. In modern terminology, we say that $A$ and $B$ are geometrically equidecomposable; this means (i) $A=\bigcup_{i=1}^{k} A_{i}$ and $B=\bigcup_{i=1}^{k} B_{i}$, (ii) $A_{i}$ and $B_{i}$ are triangles with $A_{i}{ }^{\circ} \cap A_{j}^{\circ}=B_{i}{ }^{\circ} \cap B_{j}^{\circ}=\varnothing$ for $1 \leqslant i \neq j \leqslant k$ (where $E^{\circ}$ denotes the interior of $E$ ) and (iii) $B_{i}=\tau_{i} A_{i}$ for suitable isometries $\tau_{i}, i=1, \ldots, k$, of $\mathbf{R}^{2}$. In 1924, Tarski introduced a set-theoretic version of such jig-saws. According to his definition, two sets $A$ and $B$ in $\mathbf{R}^{2}$ are equidecomposable if in the definition above we replace (ii) by (ii)' $A_{i}$ and $B_{i}$ are sets with $A_{i} \cap A_{j}=B_{i} \cap B_{j}=\varnothing$ for $1 \leqslant i \neq j \leqslant k$. In [1], Banach and Tarski proved the difficult theorem that two polygons in the plane are equidecomposable if and only if they are geometrically equidecomposable. S. Wagon's recently published book [4] is a beautiful exposition of the deep results in analysis, algebra and topology which were stimulated by [1] and other related work of the time.

In 1925, Tarski asked the still unanswered question: are a circle (with interior) and a square of the same area equidecomposable? This led G. T. Sallee to generalize the notion of geometric equidecomposability to one which can be applied to all convex bodies (compact, convex sets with nonempty interior). Two planar convex bodies $A$ and $B$ are said to be convex equidecomposable if in the definition above we replace (ii) by (ii)" $A_{i}$ and $B_{i}$ are convex bodies with $A_{i}^{\circ} \cap A_{j}^{\circ}=B_{i}^{\circ} \cap B_{j}^{\circ}=\varnothing$ for $1 \leqslant i \neq j \leqslant k$. Sallee then asked [3, Problem 1] whether Banach and Tarski's theorem can be extended to convex bodies; that is, whether two equidecomposable planar convex bodies must be convex equidecomposable (since the converse is true by the same (nontrivial) argument that Banach and Tarski used for polygons). The main purpose of this note is to answer this in the negative.

Received by the editors June 20, 1984 and, in revised form, September 17, 1984.

1980 Mathematics Subject Classification. Primary 52A10; Secondary 52A20.

$\mathrm{KeV}$. words and phrases. Convex body, equidecomposable, scissors equidecomposable, convex equidecomposable.

${ }^{1}$ Work done under University of Petroleum and Minerals Research Proposal MS/XRAYPROB/73. 
We shall include in our discussion one more type of equidecomposability. The sets $A$ and $B$ in $\mathbf{R}^{2}$ are called scissors equidecomposable if they satisfy the definition above with (ii) replaced by either (ii)* $A_{i}$ and $B_{i}$ are topological discs (images of the closed unit disc under homeomorphisms of the plane onto itself) with $A_{i}^{\circ} \cap A_{j}^{\circ}=$ $B_{i}^{\circ} \cap B_{j}^{\circ}=\varnothing$ for $1 \leqslant i \neq j \leqslant k$, or (ii) ${ }^{* *} A_{i}$ and $B_{i}$ are arcs (images of connected subsets of the circumference of a circle under homeomorphisms of the plane onto itself) such that $A_{i}$ and $A_{j}$ (and $B_{i}$ and $B_{j}$ ) meet, if at all, only on endpoints for $1 \leqslant i \neq j \leqslant k$.

In [2], it is proved that if $A$ and $B$ are elementary convex bodies which are scissors equidecomposable, then the strictly convex portions of their respective boundaries $\partial A$ and $\partial B$ are also scissors equidecomposable. Here, elementary means that the boundary consists of a finite number of arcs, each of which is either a line segment or strictly convex. In [3], Sallee uses this to show that two elementary convex bodies are convex equidecomposable if and only if they are scissors equidecomposable. He noted that a positive answer to his Problem 1 would extend the result of [2] to all convex bodies. Our answer is negative, but we shall begin by stating the appropriate extension.

Theorem 1 (Dubins, Hirsch AND Karush). Suppose $E_{1}$ and $E_{2}$ are scissors equidecomposable convex bodies in $\mathbf{R}^{2}$. Then for $i=1,2$, there are finite sets $\mathscr{L}_{i}$ of line segments in $\partial E_{i}$ such that the sets $\left(\partial E_{i} \backslash \cup \mathscr{L}_{i}\right)$ are scissors equidecomposable.

Proof. Follow [2] up to Lemma 13. Using the notation of [2], we have $E_{1}=E_{+}$ and $E_{2}=E_{-}$, and $D=D^{\prime}=E_{+}$. Also, $D^{k}$ and $D^{\prime}$ are convex bodies which are on opposite sides of the $\operatorname{arc} p(A)$. But then $p(A)$ must be a line segment, so $A$ is too. Therefore $K_{++}$consists of a finite set of line segments $\left(\mathscr{L}_{1}\right.$ above), and similarly for K.-.

Note that in Theorem 1, one cannot expect the entire boundaries $\partial E_{1}$ and $\partial E_{2}$ to be scissors equidecomposable. For, by the Wallace-Bolyai-Gerwien theorem, a square and an equilateral triangle of equal areas are scissors equidecomposable, but their boundaries have different lengths and so cannot be scissors equidecomposable.

The ideas of [3, Theorem 2] immediately give

THEOREM 2. Two planar convex bodies are convex equidecomposable if and only if they are scissors equidecomposable.

We now proceed to the examples.

THEOREM 3. There are two equidecomposable planar convex bodies which are not convex equidecomposable.

Proof. Let $C_{n}, n=1,2, \ldots$, be a sequence of disjoint closed arcs of the unit circle $C=\left\{(x, y): x^{2}+y^{2}=1\right\}$ such that each $C_{n}$ is contained in the first quadrant, $C_{n} \rightarrow\{(0,1)\}$ as $n \rightarrow \infty$, and $C_{n+1}$ lies between $C_{n}$ and the point $(0,1)$. For each $n$, suppose the endpoints of $C_{n}$ are $a_{n}$ and $b_{n}$, and let $L_{n}$ be the closed line segment $\left[a_{n}, b_{n}\right]$. We define the convex body $H$ to be the one whose boundary is $\partial H=$ $\left(C \backslash \cup_{n=1}^{\infty} C_{n}\right) \cup \cup_{n=1}^{\infty} L_{n}$. 
If $A$ is a set, let $\tau A$ be the set obtained by rotating $A$ anticlockwise by $\pi / 2$ about the origin. Denote by $K$ the convex body whose boundary is

$$
\partial K=\left[C \backslash\left(\bigcup_{n=0}^{\infty} C_{2 n+1} \cup \bigcup_{n=1}^{\infty} \tau C_{2 n}\right)\right] \cup \bigcup_{n=0}^{\infty} L_{2 n+1} \cup \bigcup_{n=1}^{\infty} \tau L_{2 n} .
$$

We show that $\partial H$ and $\partial K$ are equidecomposable, using only rotations about the origin; the equidecomposability of $H$ and $K$ follows. Let $A_{1}=\bigcup_{n=1}^{\infty} C_{2 n}, A_{2}=$ $\bigcup_{n=1}^{\infty} L_{2 n}$ and

$$
A_{3}=\left[C \backslash\left(\bigcup_{n=1}^{\infty} C_{n} \cup \bigcup_{n=1}^{\infty} \tau C_{2 n}\right)\right] \cup \bigcup_{n=0}^{\infty} L_{2 n+1} .
$$

Then $\partial H=\tau A_{1} \cup A_{2} \cup A_{3}$ and $\partial K=A_{1} \cup \tau A_{2} \cup A_{3}$, where these unions are disjoint.

Finally, we show that $H$ and $K$ are not convex equidecomposable. If they were, there would be finite sets $\mathscr{H}$ and $\mathscr{K}$ of line segments in $\partial H$ and $\partial K$ respectively, such that $(\partial H \backslash \cup \mathscr{H})$ and $(\partial K \backslash \cup \mathscr{K})$ are scissors equidecomposable. (Although this follows from Theorem 1, it is easy to see directly.) Thus there is an arc $J$ in $\partial H$ such that (i) $\bigcup_{n=N}^{\infty} L_{n} \subset J$ for some $N$, and (ii) $\partial K$ contains a congruent copy of $J$. Since (i) and (ii) are clearly contradictory, the theorem is proved.

Note that in view of Theorem 2, the equidecomposable convex bodies $H$ and $K$ above are not scissors equidecomposable.

By replacing the line segments $L_{n}$ of Theorem 3 with suitably chosen smooth, strictly convex arcs, one can show that there are even smooth and strictly convex equidecomposable planar sets which are not convex equidecomposable.

In higher dimensions, equidecomposability and convex equidecomposability are defined simply by substituting $\mathbf{R}^{n}$ for $\mathbf{R}^{2}$ in the definitions above. To deal with scissors equidecomposability, we need some definitions. A cell in $\mathbf{R}^{n}$ is the image of the sphere $S^{n-1}$ and its interior under a homeomorphism; if the homeomorphism is from $\mathbf{R}^{n}$ onto itself we call the cell tame. There are several possibilities here, but for the purposes of this paper we shall define two sets $A$ and $B$ in $\mathbf{R}^{n}(n \geqslant 3)$ to be scissors equidecomposable if condition (ii) above is replaced by (ii) ${ }^{+} A_{i}$ and $B_{i}$ are tame cells with $A_{i}^{\circ} \cap A_{j}^{\circ}=B_{i}^{\circ} \cap B_{j}^{\circ}=\varnothing$ for $1 \leqslant i \neq j \leqslant k$.

The whole nature of equidecomposability changes in $\mathbf{R}^{n}$ for $n \geqslant 3$, in view of the famous Banach-Tarski paradox. In fact [1] any two bounded sets in $\mathbf{R}^{n}(n \geqslant 3)$ with nonempty interiors are equidecomposable. Quite independently of this, our last theorem shows that Theorem 2 is no longer valid in higher dimensions. Once again, a modification will produce examples which are smooth and strictly convex.

THEOREM 4. There are two scissors equidecomposable convex bodies in $\mathbf{R}^{3}$ which are not convex equidecomposable.

Proof. The convex bodies concerned are three-dimensional versions of those in Theorem 3. On the unit sphere $S$ in $\mathbf{R}^{3}$, let $S_{n}, n=1,2, \ldots$, be a sequence of disjoint closed spherical caps with centres contained in $S$ and in the first quadrant of the $y z$-plane, such that $S_{n} \rightarrow\{(0,0,1)\}$ as $n \rightarrow \infty$ and $S_{n+1}$ lies between $S_{n}$ and $(0,0,1)$. 
Let $D_{n}$ be the flat disc with the same boundary as $S_{n}$. Then $H^{\prime}$ is the convex body whose boundary is $\partial H^{\prime}=\left(S \backslash \cup_{n=1}^{\infty} S_{n}\right) \cup \cup_{n=1}^{\infty} D_{n}$, and $K^{\prime}$ is the convex body such that

$$
\partial K^{\prime}=\left[S \backslash\left(\bigcup_{n=0}^{\infty} S_{2 n+1} \cup \bigcup_{n=1}^{\infty} \tau S_{2 n}\right)\right] \cup \bigcup_{n=0}^{\infty} D_{2 n+1} \cup \bigcup_{n=1}^{\infty} \tau D_{2 n},
$$

where $\tau$ now denotes anticlockwise rotation about the origin by $\pi / 2$ in the $y z$-plane.

It will suffice to show that $H^{\prime}$ and $K^{\prime}$ are scissors equidecomposable, since the proof that they are not convex equidecomposable is analogous to that in Theorem 3. Let $T_{1}$ be a tame cell contained in $S$ and its interior and also in the half-space $\{y$ : $y \geqslant 0\}$, such that $T_{1} \cap S=\cup_{n=1}^{\infty} S_{2 n} \cup\{(0,0,1)\}$. Let $T_{2}$ be the tame cell such that $\partial T_{2}=\left(\partial T_{1} \backslash \cup_{n=1}^{\infty} S_{2 n}\right) \cup \bigcup_{n=1}^{\infty} D_{2 n}$. Lastly, let

$$
V=\operatorname{cl}\left(H^{\prime} \backslash\left(\tau T_{1} \cup T_{2}\right)\right)=\operatorname{cl}\left(K^{\prime} \backslash\left(T_{1} \cup \tau T_{2}\right)\right),
$$

and put $T_{3}=V \cap\{x: x \geqslant 0\}$ and $T_{4}=V \cap\{x: x \leqslant 0\}$. It is not difficult to see that $T_{3}$ and $T_{4}$ are tame cells. Now $H^{\prime}=\tau T_{1} \cup T_{2} \cup T_{3} \cup T_{4}$ and $K^{\prime}=T_{1} \cup \tau T_{2} \cup$ $T_{3} \cup T_{4}$, where the sets in each of these unions have disjoint interiors. This completes the proof.

\section{REFERENCES}

1. S. Banach and A. Tarski, Sur la décomposition des ensembles de points en parties respectivement congruentes, Fund. Math. 6 (1924), 244-277.

2. L. Dubins, M. Hirsch and J. Karush, Scissor congruence, Israel J. Math. 1 (1963), 239-247.

3. G. T. Sallee, Are equidecomposable plane convex sets convex equidecomposable? Amer. Math. Monthly 76 (1979), 926-927.

4. S. Wagon, The Banach-Tarski paradox, Cambridge Univ. Press, New York, 1985.

Department of Mathematical Sciences, University of Petroleum and Minerals, Dhahran, Saudi Arabia 